\title{
Multiple autoimmune syndrome with celiac disease
}

\author{
Singh Harpreet, Jain Deepak, B. Kiran \\ Pandit Bhagwat Dayal Sharma University of Health Sciences, Rohtak (Haryana), India
}

\begin{abstract}
Multiple autoimmune syndrome (MAS) is a condition characterised by three or more autoimmune disorders in a same individual. Familial, immunologic and infectious factors are implicated in the development of MAS. Here we report a case of a 32-year-old woman with co-existence of four autoimmune diseases, namely autoimmune hypothyroidism, Sjögren's syndrome, systemic lupus erythematosus (SLE) and celiac disease which leads to the final diagnosis of multiple autoimmune syndrome type 3 with celiac disease. Patients with single autoimmune disorder are at $25 \%$ risk of developing other autoimmune disorders. The present case emphasises to clinicians that there is a need for continued surveillance for the development of new autoimmune disease in predisposed patients.
\end{abstract}

Key words: multiple autoimmune syndrome, Sjögren's syndrome, systemic lupus erythematosus, celiac disease.

\section{Introduction}

Autoimmune diseases are the conditions initiated by the loss of immunological tolerance to self-antigens and that make up, a heterogeneous group of disorders in which multiple alterations in the immune system result in a group of syndromes that either target specific organs or affect the body systematically [1]. Multiple autoimmune syndrome (MAS) is defined as occurrence of at least three autoimmune diseases in a same patient [2]. It is further classified into type 1 MAS, type 2 MAS and type 3 MAS. Celiac disease affects $1 \%$ of the general population and is an important autoimmune disease, but it is not included in the classification of MAS. As per literature evidence celiac disease is strongly associated with autoimmune hypothyroidism and Sjögren's syndrome in $2-7 \%$ and $4.5-15 \%$ respectively $[3,4]$ and is very less commonly associated with systemic lupus ewrythematorus (SLE). It is of interest, the presence of one autoimmune disorder makes a path to discover the other autoimmune conditions and improving their therapeutical measures. The presented case shows clinicians that there is a need for continuous surveillance for the development of other autoimmune diseases in predisposed patients have already diagnosed with autoimmune disease.

\section{Case report}

The 32-year-old female patient was directed to our hospital with complaints of intermittent pain in multiple symmetrical joints accompanied by morning stiffness for the past 5 years which involved small joints of hands, wrists and knees. However, there was no history of any joint swelling. The patient also complained of chronic fatigue for the past 3 years. The patient had been diagnosed with hypothyroidism and had been treated with thyroxine $100 \mu \mathrm{g} /$ day for the last 2 years. Also there was a history of loose stools on and off since 2 years, dryness of eyes and mouth for 6 months and photosensitivity for the last year. She has two children and the last child birth was 5 years ago. Both deliveries were uneventful. There was no history of any miscarriages and she had regular menstrual cycles with no history of menorrhagia or dysmenorrhoea. There was no history of any other chronic illness or chronic drug intake. Family history and personal history were not significant.

\footnotetext{
Address for correspondence

Jain Deepak, Department of Medicine, Pt B D Sharma University of Health Sciences, 124001 Rohtak, India,

e-mail: jaindeepakdr@gmail.com

Submitted: 9.10.2016, Accepted: 9.12.2016
} 
On general physical examination, the patient was moderately built, conscious and oriented to time place and person. Vitals signs were within the normal limits. She was pale and there was no icterus, cyanosis, clubbing, koilonychia, lymphadenopathy or pedal oedema. There was no swelling in the anterior aspect of neck. She has mild pain on movement of wrist and knee joint however there was no joint oedema, deformity or restriction of mobility. Clinical examinations of respiratory, abdominal, cardio-vascular and nervous system were unremarkable.

Complete hemogram revealed haemoglobin of $8.2 \mathrm{~g} \%$ with microcytic hypochromic picture. White blood count and platelet count were normal. Renal function tests, liver function tests with serum proteins with $A$ : G ratio, Blood glucose, serum electrolytes lipid profile were normal. HIV, HbsAg, anti HCV antibody tests were non-reactive. Stool examination for occult blood was negative. Urine complete examination showed albuminuria with 24 hour urinary protein of $1.2 \mathrm{~g}$ and there was no dysmorphic RBCs, leukocytes, or any urinary casts. Schirm- er's test was positive for dryness symptoms with $3 \mathrm{~mm}$ (right eye) and $2 \mathrm{~mm}$ (left eye). DEXA scan showed Bone density less than expected for the age group. The ECG, chest X-ray and 2D echocardiography showed no abnormalities. Ultrasound imaging of thyroid showed bilateral lobes altered echo texture and increased flow on colour Doppler, USG abdomen showed few small renal calculi in right kidney. All other lab investigations are summarised in Table I.

In view of IgA tTG positivity and chronic diarrhoea Upper Gl Endoscopy was done and biopsy was taken which showed ulcerations in mucosa and increase in intraepithelial lymphocytes (> 40 lymphocytes/100 enterocytes) with lamina propria showing inflammatory infiltrate comprising lymphocytes, plasma cells and eosinophils and complete loss of villi findings suggestive of celiac disease (modified marsh grade III) (Fig. 1). Renal biopsy was also done which was normal (Fig. 2).

Based on the history, clinical findings and afore mentioned laboratory investigations which indicated the coexistence of celiac disease with autoimmune hypo-

Table I. Special laboratory investigations

\begin{tabular}{|c|c|}
\hline Investigations & Results \\
\hline Serum calcium concentration & $8.2 \mathrm{mg} / \mathrm{dl}(\mathrm{n}: 9-10.5 \mathrm{mg} / \mathrm{dl})$ \\
\hline Serum phosphate concentration & $2.4 \mathrm{mg} / \mathrm{dl}(\mathrm{n}: 2.5-4.5 \mathrm{mg} / \mathrm{dl})$ \\
\hline Blood pH & $7.30(\mathrm{n}: 7.35-7.45)$ \\
\hline Bicarbonate $\left(\mathrm{HCO}_{3}\right)$ & $15.6 \mathrm{mEq} / \mathrm{l}(\mathrm{n}: 22-30 \mathrm{mEq} / \mathrm{l})$ \\
\hline Serum sodium concentration & $141 \mathrm{mmol} / \mathrm{l}(\mathrm{n}: 135-145 \mathrm{mmol} / \mathrm{l})$ \\
\hline Serum potassium concentration & $2.3 \mathrm{mmol} / \mathrm{l}(\mathrm{n}: 3.5-5.0 \mathrm{mmol} / \mathrm{l})$ \\
\hline ESR (erythrocyte sedimentation rate) & 66 mm/h (n: 0-20 mm/h) \\
\hline C-reactive protein & $6 \mathrm{mg} / \mathrm{l}(\mathrm{n}: 0-5 \mathrm{mg} / \mathrm{l})$ \\
\hline Urinary $\mathrm{pH}$ & $6.5(n: 5-9)$ \\
\hline Triiodothyronine (T3) & 0.42 (n: 0.60-1.81 ng/dl) \\
\hline Thyroxine (T4) & 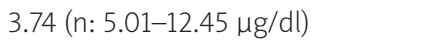 \\
\hline Thyroid-stimulating hormone (TSH) & $7.36 \mu \mathrm{lU} / \mathrm{ml}(\mathrm{n}: 0.5-5.0 \mu \mathrm{lU} / \mathrm{ml})$ \\
\hline Anti-thyroperoxidase (TPO) antibody & $1230(\mathrm{n}:<35 \mathrm{IU} / \mathrm{l})$ \\
\hline ANA (indirect immunofluorescence assay) & Positive, 1 : 1280, Speckled pattern \\
\hline Anti-dsDNA antibody & $1.59 \mathrm{Al}(\mathrm{n}<0.9 \mathrm{Al})$ \\
\hline Anti-Ro antibody & $10.78 \mathrm{IU} / \mathrm{ml}(\mathrm{n}<3 \mathrm{IU} / \mathrm{ml})$ \\
\hline Anti-La antibody & $9.17 \mathrm{IU} / \mathrm{ml}(\mathrm{n}<3 \mathrm{IU} / \mathrm{ml})$ \\
\hline P-anti-neutrophil cytoplasmic antibody & Negative \\
\hline C-anti-neutrophil cytoplasmic antibody & Negative \\
\hline Complement component 3 (C3) & 99.40 (n: 90-180 mg/dl) \\
\hline Complement component 4 (C4) & 21.50 (n: 10-40 mg/dl) \\
\hline IGA tissue transglutaminase (tTG) antibody & $181 \mathrm{IU} / \mathrm{ml}(\mathrm{n}:<25 \mathrm{IU} / \mathrm{ml})$ \\
\hline Rheumatoid factor & Negative \\
\hline
\end{tabular}




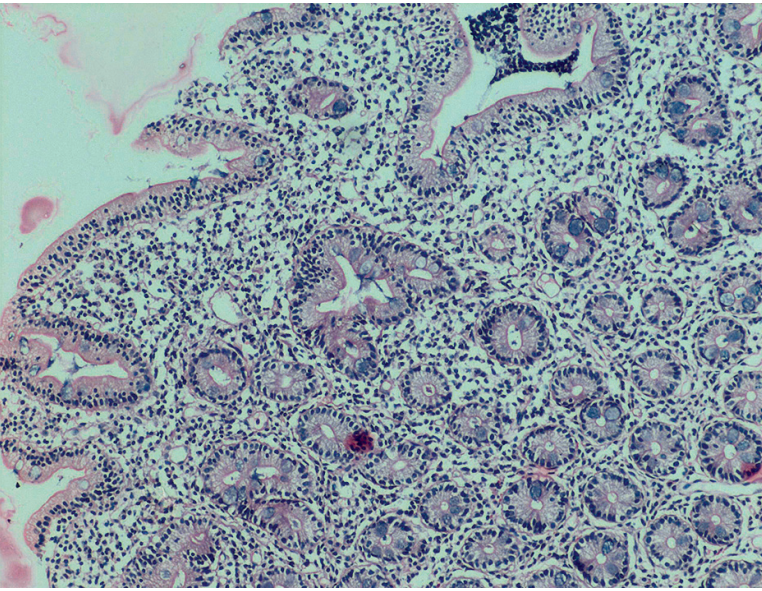

Fig. 1. Histopathological picture of gut biopsy (celiac disease).

thyroidism, Sjögren's syndrome (Revised International Classification Criteria for Sjögren's Syndrome), and systemic lupus erythematosus (SLICC Revised Criteria) and thus the diagnosis was concluded as celiac disease with MAS type 3. The patient was continued with thyroxine and was put on gluten free diet, short course steroids, lubricant eye drops, tab pilocarpine, potassium rich diet and oral potassium supplements. She improved gradually and is under regular follow up.

\section{Discussion}

Multiple autoimmune syndrome is a condition with coexistence of three or more autoimmune disease in a single patient. It is further classified into MAS type 1 , 2 and 3 as described in Table II. This classification is important in detecting a new disease entity liable to appear in a patient who has had prior autoimmune diseases. It gives a basis for analysis of the pathophysiology of autoimmunity. The pathogenesis of multiple autoimmune disorders is not clearly understood but certain environmental factors are believed to cause disorders of immune regulation in genetically predisposed individuals.

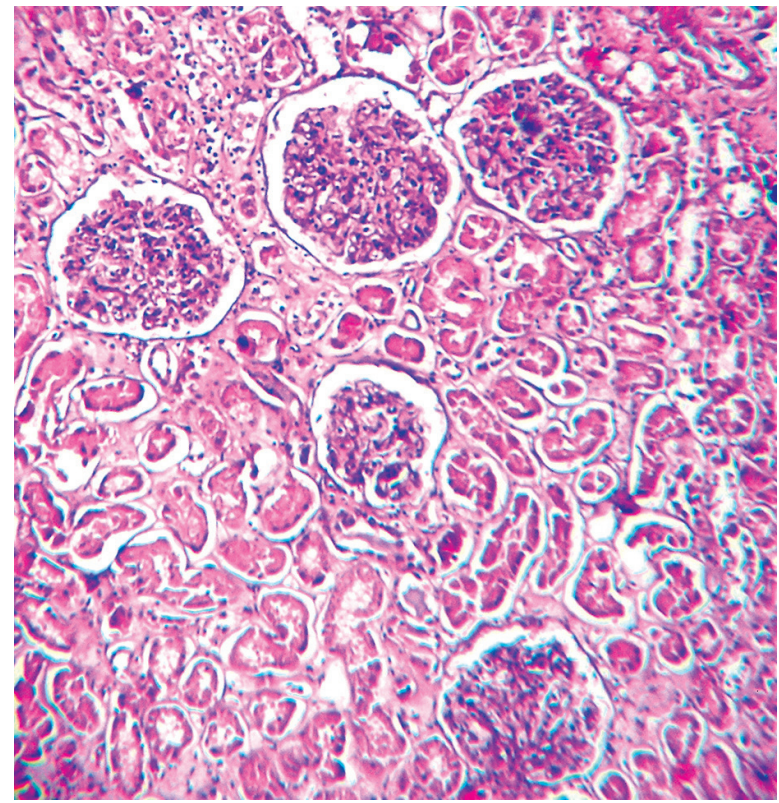

Fig. 2. Histopathological picture of renal biopsy (normal).

Multiple auto antibodies can be found in a patient and some of the specific mono- or polyclonal autoantibodies may be multiple organ reactive. Researchers note that in many cases, the presence of one autoimmune disorder leads to discovery of other autoimmune conditions.

In the case of the described patient with autoimmune hypothyroidism with positive TPO antibody we looked into other auto immune diseases based on her symptoms. The patient was IgA tTG positive and her duodenal biopsy was suggestive of celiac disease. Evidences shows that in patients with celiac disease occurrence of thyroid dysfunction is up to $10 \%$ of cases, and risk of thyroid disease is threefold higher. The coexistence of autoimmune thyroid disease and celiac disease is thought to be due to a common genetic predisposition. Inheritance of HLA-DQ2 and DQ8 haplotypes which are over-represented in many autoimmune diseases and

Table II. Types of multiple autoimmune syndrome

\begin{tabular}{|ccc|}
\hline Type 1 MAS & Type 2 MAS & Type 3 MAS \\
\hline Myasthenia gravis & Sjögren's syndrome & Sjögren's syndrome \\
\hline Thymoma & SLE & Myasthenia gravis and or thymoma \\
\hline Polymyositis & Rheumatoid arthritis & Addison's disease \\
\hline Giant cell myocarditis & Primary biliary cirrhosis & Type 1 diabetes mellitus \\
\hline & Scleroderma & Autoimmune haemolytic anaemia, Pernicious \\
& & anaemia, ITP \\
\hline & Autoimmune hypothyroidism & Autoimmune hypothyroidism \\
\hline & & Dermatitis herpetiformis \\
\hline
\end{tabular}


which are associated immunological phenotype may explain the link [5].

The patient also had symptoms suggestive of Sjögren's syndrome and distal renal tubular acidosis (RTA) which was later on confirmed by Schirmer's test, anti Ro and anti La antibodies along with urinary $\mathrm{PH}$, blood gas analysis and renal calcinosis. The co-existence of Sjögren's syndrome and autoimmune thyroiditis is frequent and suggests a common genetic or environmental factor predisposition with similar pathogenic mechanisms. Sjögren's syndrome was 10 times more frequent in patients with autoimmune thyroid disease, and autoimmune thyroiditis was 9 times more frequent in Sjögren's syndrome [6]. Studies show that $15 \%$ of cases diagnosed with Sjögren's syndrome also have biopsy proven celiac disease meaning that celiac disease is far more common in Sjögren's syndrome patients than in the general population [4].

The patient had features of SLE (oral ulcers, synovitis, photosensitivity, proteinuria) with presence of anti-nuclear antibodies (ANAs), anti-dsDNA antibodies positive and SLICC criteria for classification of SLE were met. Estimated prevalence of Sjögren's syndrome with in course of SLE is about $14.8 \%$. Studies have found that people with SLE and secondary Sjögren's overlap are older at the time of diagnosis are more likely to have joint pain, rashes; however they have a lower risk of developing kidney involvement due to SLE $[7,8]$. Approximately $15-30 \%$ of patients with SLE have anti-thyroid antibodies while $6 \%$ had actual hypothyroidism.There are reports of patients with SLE having the anti-gliadin autoantibodies but no evidence of celiac disease on biopsy $[9,10]$. Finally these four autoimmune disease in case of described patient leads to diagnosis of multiple autoimmune syndrome type 3 with celiac disease.

\section{Summary}

Patients with a single autoimmune disorder are at $25 \%$ risk of development other autoimmune disorders [10]. The possibility of three or more autoimmune diseases occurring in the same patient cannot be fortuitous and suggests a pathogenic relationship between each of them. Presence of one autoimmune disease should elicit vigilance for another one. Occurrence of multiple autoimmune phenomena indicates the need for continued surveillance for the development of new autoimmune disease in predisposed patients. Early identification of the individual disease of the MAS is needed in order to decrease the morbidity and mortality associated with MAS.

\section{References}

1. Anaya JM, Shoenfeld Y, Correa PA, et al. Autoimmunity and Autoimmune Disease. CIB, Medellin 2005.

2. Humbert P, Dupond JL. Multiple autoimmune syndromes. Ann Med Interne (Paris) 1988; 139: 159-168.

3. Spadaccino AC, Basso D, Chiarelli S, et al. Celiac disease in North Italian patients with autoimmune thyroid diseases. Autoimmunity 2008; 41: 116-121.

4. Liden M, Kristjánsson G, Valtýsdóttir S, Hällgren R. Gluten sensitivity in patients with primary Sjögren's syndrome. Scand J Gastroenterol 2007; 42: 962-967.

5. Green JR, Chiew MK, Low HC, Woodrow JC. The association between HLA antigens and the presence of certain diseases. Stat Med 1983; 2: 79-85.

6. Baszis K, Toib D, Cooper M, et al. Recurrent parotitis as a presentation of primary pediatric Sjögren syndrome. Pediatrics 2012; 129: 179-182.

7. Manoussakis MN, Georgopoulou C, Zintzaras E, et al. Sjögren's syndrome associated with systemic lupus erythematosus: clinical and laboratory profiles and comparison with primary Sjögren's syndrome. Arthritis Rheum 2004; 50: 882-891.

8. Baer AN, Maynard JW, Shaikh F, et al. Secondary Sjögren's syndrome in systemic lupus erythematosus defines a distinct disease subset. J Rheumatol 2010; 37: 1143-1149.

9. Rensch MJ, Szyjkowski R, Shaffer RT, et al. The prevalence of celiac disease autoantibodies in systemic lupus erythematosus. Am J Gastroenterology 2001; 96: 1113-1115.

10. Mohan MP, Ramesh TC. Multiple autoimmune syndrome. Indian J Dermatol Venereol Leprol 2003; 69: 298-299.

The authors declare no conflict of interest. 